\title{
PROGRAMAS DE TRANSFERENCIAS MONETARIAS CONDICIONADAS EN MÉXICOY CHILE: DISTRIBUCIÓN Y MODELO PROBIT DE LA POBREZA
}

\author{
CONDITIONAL CASH TRANSFER PROGRAMS IN MEXICO AND CHILE: \\ DISTRIBUTION AND PROBIT MODEL OF POVERTY**
}

\author{
María del Carmen Hernández Eguiarte $e^{(* *)}$ \\ Escuela Superior de Economía del Instituto Politécnico Nacional, México (DF) \\ Genaro Aguilar Gutiérrez $z^{(* * *)}$ \\ Universidade Estadual de Campinas, São Paulo, Brasil
}

Resumen: Los Programas de Transferencias Monetarias Condicionadas (PTC) son el eje rector de la política social para el combate contra la pobreza en América Latina, como en el caso de México con el programa Oportunidades y Chile mediante el sistema Chile Solidario. Con microdatos de encuestas de los hogares de México (2012) y Chile (2011), se calcula el grado de concentración de las transferencias y un modelo probit que busca definir su efecto sobre la probabilidad de ser pobre. El hallazgo principal es que la pertenencia a dichos programas no disminuye la probabilidad de ser pobre, un resultado contrario al deseado.

Palabras clave: Pobreza en México y Chile; Programas de Transferencias Monetarias Condicionadas; Modelo Probit de la pobreza.

\begin{abstract}
The Conditional Cash Transfer Programs (CCT) are the guiding principle of social policy in Latin America. Mexico, with Oportunidades Program and Chile with Chile Solidario face the phenomenon of poverty they have. Using microdata from household surveys for Mexico (2012) and Chile (2011), we calculated the degree of concentration of transfers and also estimate a probit model that seeks to define the effect of the programs on the probability of being poor. The main finding is that Oportunidades and Chile Solidario have an opposite effect; the membership in the program does not reduce the probability of being poor.
\end{abstract}

Keywords: Poverty in Mexico and Chile; Conditional Cash Transfers Programs; Probit model of poverty.

(*) Este artículo es resultado del proyecto de investigación denominado "Desarrollo Económico con Inclusión Social: Política Social para grupos vulnerables" con número de registro 20140085 de la Secretaría de Investigación y Posgrado del Instituto Politécnico Nacional, México.

(**) Maestra, Estudiante del Programa de Doctorado en Ciencias Económicas en la especialidad de Desarrollo Económico de lo Instituto Politécnico Nacional. E-mail: <mche_1988@hotmail.com>.

${ }^{(* * *)}$ Doctor, Profesor Titular C del en el posgrado en Ciencias Económicas del Instituto Politécnico Nacional. E-mail: <gaguilarg@ipn.mx>. Recibido en: 20.03.2014, aceptado en: 04.02.2015. 


\section{INTRODUCCIÓN}

La pobreza se ha convertido en un problema al que se le otorga prioridad en el manejo de la política pública, por lo que los gobiernos han diseñado Programas de Transferencias Monetarias Condicionadas (PTC), siendo América Latina pionera en su aplicación. El objetivo de estos programas es fomentar la demanda de bienes y servicios mediante un ingreso condicionado a un conjunto de corresponsabilidades en términos de acumulación de capital humano (STAMPINI y TORNAROLLI, 2012; CECCHINI y MADARIAGA, 2011; CECCHINI y MARTÍNEZ, 2011; BM, 2010; FISZBEIN y SCHADY, 2009; PEREZ, 2008; VILLATORO, 2008; CEPAL, 2006; VILLATORO, 2006; LEVY y RODRÍGUEZ, 2005; RAVALLION, 2003; RAWLINGS y RUBIO, 2003). La idea del mantenimiento de un nivel de ingreso base surge por el Plan de Seguridad Social contenido en el Reporte de Beveridge desarrollado a mediados del siglo XX (WOLMAN, 1943; BURNS, 1943; BEVERIDGE, 1942), que significó el punto de partida para el establecimiento de esquemas de seguridad social y programas de cobertura nacional (MYLES y QUADAGNO, 2002).

Autores destacados sobre el diseño e impacto de los PTC son: Schaffland (2012), Hoces y Hojman (2011), Angelucci (2012), Rivera (2009), Arriagada y Mathievet (2007), Rawlings y Rubio (2003). El protagonismo de los PTC en la política social es cuestionado dado el creciente nivel de pobreza que caracteriza a las naciones. México y Chile son dos países emblemáticos en el tema con sus respectivos programas: Oportunidades y Chile Solidario, sobre los cuales descansará este trabajo. Por tanto, es necesario determinar el efecto que estos programas tienen en la superación de la pobreza, específicamente en la probabilidad de ocurrencia de la misma.

La estructura del documento es la siguiente: en la sección 2, se describen los aspectos teóricos y evidencia empírica del fenómeno de pobreza, así como los programas principales para su combate en México y Chile. La sección 3 contiene la metodología aplicada para el cálculo del índice de concentración y el modelo probit de la pobreza, cuyos resultados se muestran en la sección 4 y por último, en la sección 5 , se presentan las conclusiones del trabajo.

\section{EVOLUCIÓN DE LA POBREZA Y PTC: MÉXICO Y CHILE}

En términos de historia de seguridad social, The Social Insurance and Allied Services Report escrito por Sir William Beveridge marca el inicio de un nuevo enfoque de asistencia social alrededor del mundo (FREDERICKS, 1978; WOLMAN, 1943). La columna vertebral del Reporte de Beveridge era el aseguramiento y mantenimiento de un nivel de ingreso necesario para la subsistencia, el cual era posible mediante la cooperación entre el Estado y el individuo (BURNS, 1943; WOLMAN, 1943; BEVERIDGE, 1942). Por la influencia de este reporte y la creciente importancia que ha cobrado la pobreza en el manejo de la política pública, muchos países han establecido esquemas de seguridad social y programas de cobertura nacional.

La pobreza se refiere a un estado de carencia de los medios para atender las necesidades fundamentales, principalmente aquellas referidas al sustento de la vida (BOLT- 
VINIK y HERNÁNDEZ, 2000). El combate a la pobreza se logra mediante una política social que se vale de los planes del gobierno, concretizados en programas sociales aplicables en todos los niveles, para alcanzar el bienestar y seguridad social de las familias (RANGEL, 2010; LEVY y RODRÍGUEZ, 2005).

Respecto a la medición de la pobreza, el método de Líneas de Pobreza (LP) se define como el indicador por excelencia, por lo que al aplicarlo un número importante de países es posible la comparación de las cifras. De acuerdo con Ravallion (1998), la línea de pobreza se define como el costo monetario de una determinada persona, en un lugar y tiempo dado, del nivel de referencia de bienestar. Expresado de otra forma, la línea de pobreza se define como el ingreso monetario per cápita necesario para adquirir los bienes y servicios básicos que permitan el acceso a un nivel de vida adecuado. Si el ingreso de una determinada persona está por debajo de dicho nivel, se considerará pobre, de lo contrario no pertenecerá al umbral de la pobreza (CETRÉ, 2006; FERES, 2001).

Bajo el método de LP, cuya fuente principal son las encuestas de ingresos de los hogares, se presenta a continuación la definición de la pobreza en México y Chile.

Para el caso mexicano, se tienen las siguientes categorías:

- Pobreza alimentaria: Se encuentra en esta situación aquella persona que posee un ingreso per cápita insuficiente para acceder a una alimentación mínimamente aceptable.

- Pobreza de capacidades: Se encuentra en esta situación aquella persona que si bien puede cubrir sus necesidades mínimas de alimentación, cuenta con un ingreso per cápita insuficiente como para realizar las inversiones mínimamente aceptables en educación y salud.

- Pobreza de patrimonio: Se encuentra en esta situación aquella persona que si bien puede cubrir sus necesidades mínimas de alimentación, salud y educación, cuenta con un ingreso per cápita insuficiente como para realizar las inversiones en vestido, calzado, vivienda y transporte público (De la Vega, 2010; PND 2007-2012).

En Chile, las clasificaciones de la pobreza que se calculan son:

- Pobreza extrema (indigencia): Considera las personas cuyo ingreso es insuficiente para satisfacer sus necesidades alimentarias; esto es, que pertenecen a hogares cuyos ingresos no son suficientes para costear una canasta básica de alimentos a sus miembros.

- Pobreza: Se refiere a las personas cuyo ingreso es insuficiente para satisfacer sus necesidades básicas; esto es, que pertenecen a hogares cuyos ingresos no son suficientes para satisfacer las necesidades básicas de sus miembros (Mideplan, 2013).

En México, en el año 1996 la pobreza alcanzó su máximo nivel con tasas de crecimiento (respecto a 1994) de $82.3,61.6$ y 36 por ciento para pobreza alimentaria, de capacidades y patrimonial respectivamente. Estas tasas de crecimiento están asociadas a 
la crisis iniciada en 1995, año en el cual el PIB per cápita en todo el país cayó 10.2\%. En años posteriores, la pobreza cayó, entre otros factores, por el aumento de las remesas y el inicio del Programa de Educación, Salud y Alimentación (Progresa). La disminución del nivel de pobreza sólo ocurrió hasta el año 2006, a partir del cual ha incrementado nuevamente, siendo en 2008 el aumento de 37.2, 25.7 y 14.7 por ciento para cada categoría. En 2012, el 19.7, 28 y 52.3 por ciento de la población se encontraba en situación de pobreza de acuerdo a la clasificación oficial, lo que equivale a 23, 32 y 61 millones de personas. Estos aumentos están asociados a la crisis mundial iniciada en 2008, que tuvo un impacto negativo importante sobre las remesas.

En Chile, a partir de 1992, las cifras de pobreza han registrado disminuciones de hasta dos dígitos como el caso de 1994 y 2006 , que son de 13 y 22.3 por ciento respectivamente; sin embargo, para los años 2009 y 2011, la pobreza ha aumentado en 11.6 y 4.6 por ciento, lo que en términos absolutos equivale a 1.8 y 1.9 millones para cada año. Respecto a la pobreza extrema, las reducciones registradas en la mayoría de los años han sido mayores a las tasas de crecimiento registradas en 1998, 2000 y 2009 de $0.4,1.5$ y 20.1 por ciento respectivamente. En 2011, se registró una disminución de casi 24 puntos porcentuales lo que condujo en términos absolutos a 472 mil personas en situación de indigencia.

Para combatir este problema social, ambas naciones han recurrido a los Programas de Transferencias Monetarias Condicionadas como principal instrumento, cuyo objetivo "declarado" es romper el ciclo intergeneracional de la pobreza a través de la inversión de capital humano.

Pero, ¿Por qué surgen estos programas? ¿Qué hay detrás de ellos? ¿Cuál es el contexto político que está detrás del surgimiento de los Programas de Transferencias condicionadas de Ingreso, tanto en México como en Chile? Como se sabe, a partir de los años 90 ambos países, en respuesta a la crisis de la deuda de los 80, emprendieron sendas políticas de ajuste y cambio estructural. Estas políticas de ajuste y cambio estructural inspiradas en el decálogo del Conseso de Washington, en el terreno social dejaban de "manos atadas" a grandes segmentos de la población: se instaura el mainstrem. Se abandonaron los antiguos y robustos sistemas de Protección Social masivos. Es decir, al postular el retiro del Estado de la Economía y el adelgazamiento de las políticas públicas; en el terreno social el resultado fue reducir la intervención del Estado (subsidios a la oferta, inversiones en educación, salud, vivienda y sistemas de alimentación amplios, etc.) a una mera transferencia de ingreso hacia los más pobres; evitando así los "onerosos" gastos que representaban mantener un gran Estado con alcance e intervención hacia todas las esferas de lo social.

Los Programas de Transferencias monetarias condicionadas surgen, así, como un mero "paliativo", ante el desmantelamiento de los antiguos Sistemas de Protección Social emanados de la segunda posguerra.

\subsection{MÉXICO: PROGRAMA DE DESARROLLO HUMANO OPORTUNIDADES}

Las crecientes cifras de pobreza registradas en el país durante la crisis de 1995 demandaron un cambio de estrategia en la política de combate a la pobreza extrema 
en México, por lo que en 1997 se lanza el Programa de Educación, Salud Alimentación (Progresa). La creación de Progresa fue el resultado de un esfuerzo conjunto entre las Secretaría de Educación, de Salud y de Hacienda y Crédito Público, además de que procedió de un amplio e intenso debate en los tres órdenes de gobierno, partidos políticos y grupos de interés. Desde el planteamiento inicial hasta el lanzamiento del programa transcurrieron dos años, concentrándose en 1997 en localidades rurales con menos de 2500 habitantes que se encontraban en situación de extrema pobreza. La cobertura inicial fue de 300,000 hogares, incrementando a 2.5 millones de familias para el año 2000, a las que se les otorgaban: transferencias en efectivo, suplementos alimenticios y acceso a un paquete básico de servicios de salud, con la condición de que se cumpliera un conjunto de requisitos en los ámbitos de nutrición, salud y educación (STAMPINI y TORNAROLLI, 2012; FISZBEIN y SCHADY, 2009; SOARES, 2009; VILLATORO, 2006; LEVY Y RODRÍGUEZ, 2005).

En 2001, el nombre del programa se cambió a Oportunidades y su cobertura se expandió a localidades urbanas, que contenían de 2500 a 14999 habitantes. Para el año 2002, todas las áreas fueron cubiertas, lo que resultó en 5 millones de hogares beneficiados para 2004 y 5.8 millones para 2010 (HERNÁNDEZ, 2012; SOARES, 2009). Un cambio adicional en la transición de Progresa a Oportunidades fue que se añadió un componente denominado Jóvenes con Oportunidades, que aumentó las transferencias de ingreso a las familias pobres y cuya población objetivo son los jóvenes que cursan desde tercero de secundaria hasta tercer año del nivel medio superior (LEVY y RODRÍGUEZ, 2005). Mediante la operación del programa y bajo la metodología de los PTC, el gobierno mexicano pretender alcanzar la meta de erradicar la pobreza para el año 2030 (SEDESOL, 2013).

\subsection{CHILE: SISTEMA CHILE SOLIDARIO}

El antecedente del Sistema Chile Solidario es el Subsidio Unitario Familiar, el cual comenzó en la década de los 80 con el objetivo de fomentar el desarrollo de capital humano mediante la educación y el empleo, sin el requisito de condicionalidades que garantizaran o promovieran la participación activa de los beneficiarios (Fiszbein y Schady, 2009).

El sistema Chile Solidario, y su componente principal, el Programa Puente, se crea en el año 2002 como parte del Sistema de Protección Social, encargado de atender a la población más vulnerable del país y como resultado de un difícil proceso de pobreza que no había disminuido durante los años noventa (MIDEPLAN, 2013; FISZBEIN Y SCHADY, 2009; SOARES, 2009).

Chile Solidario se define como un sistema descentralizado que funciona en coparticipación con gobiernos de todos los órdenes, los cuales son los encargados de la protección social de la población. La participación del sistema en la política social para combatir la pobreza se fortalece aportando recursos técnicos, metodológicos y financieros que aseguren la atención oportuna y de calidad a las familias más vulnerables dentro del territorio chileno. El alcance de los objetivos se logra mediante la coparticipación de programas propios de la política social, funcionando además como 
dispositivo de intermediación, consejería y acompañamiento para el tratamiento y solución del problema de pobreza existente.

A pesar de la reducción de la pobreza por concepto del Sistema Chile Solidario, durante un periodo, las cifras se estancaron, lo cual fue resuelto mediante la creación de un sistema de apoyo psicosocial que permitió derribar la barrera contra la que se enfrentaba el programa (CECCHINI y MARTÍNEZ, 2011), una característica muy distintiva en el desarrollo de los programas sociales en Chile.

\section{METODOLOGÍA}

Las encuestas de los hogares proveen una rica fuente de datos sobre el comportamiento económico y sus vínculos con la política pública. La información que proporcionan a nivel de los individuos de los hogares ofrece indicios de la situación general (o específica) prevaleciente de un país, además, por su diseño e implementación, se definen como la fuente principal para el cálculo de pobreza bajo la metodología de Líneas de Pobreza, en donde el ingreso es el indicador sobre el cual se realizan las estimaciones (DEATON, 1997). Por lo anterior, los microdatos de las encuestas de los hogares serán la fuente de información para este trabajo: en el caso de México la Encuesta Nacional de Ingresos y Gastos de los Hogares (ENIGH) 2012 y para Chile, la Encuesta de Caracterización Socioeconómica Nacional (CASEN) 2011.

\section{1 ÍNDICE DE CONCENTRACIÓN}

El índice de concentración de los programas sociales permite definirlos como progresivos o regresivos, es decir, saber si las transferencias asignadas se concentran en los primeros deciles de ingreso, lo que es ideal dado su diseño y objetivos. La obtención de los índices de concentración se realiza siguiendo la premisa de Fei (1978), quien reconoce que el patrón de ingreso total de $\mathrm{n}$ familias tiene un número finito de $\mathrm{r}$ componentes del mismo $W^{i}=\left(W_{1}^{i}, W_{2}^{i}, \ldots, W_{n}^{i}\right)(i=1,2, \ldots, r)$. Donde $\mathrm{Y}$ es el vector de suma de, compuesto por salario, transferencias gubernamentales entre otras fuentes de ingreso.

En el caso de México, se calcula el índice de concentración del programa Oportunidades y para el caso de Chile, dado que el Sistema Chile Solidario posee múltiples componentes, se calcula con las transferencias de Asignación Social y Bono de Protección Familiar y de Egreso. En el caso de Asignación Social, su metodología se rige bajo el concepto de los PTC, una asignación de un monto per cápita sujeta a un conjunto de condicionalidades así como al cumplimiento de los logros que permitan la superación del estado de pobreza. Por otro lado, el ingreso por Bono de Protección Familiar y de Egreso se otorga a las familias en extrema pobreza que pertenezcan al Programa Puente, definido como otro de los componentes del Sistema Chile Solidario y que funciona bajo la misma metodología de los PTC.

\subsection{MODELO PROBIT DE LA POBREZA}

El modelo Probit permite explicar el comportamiento de una variable dependiente binaria mediante la implementación de una función de distribución acumulativa nor- 
mal. La distribución logística normal estándar de esta clase de modelos se caracteriza por tener media cero y varianza igual a 1 (GUJARATI y PORTER, 2010; GREENE, 1998).

La especificación funcional de los modelos Probit aplicados aquí es la siguiente:

$$
\text { poblp3 }(\text { poblp })=\beta_{0}+\beta_{i 1} C S E+\beta_{i 2} C V+\beta_{i 3} C O L+\beta_{i 4} O V+\beta_{i 5} P T C+U_{i} \text { (1) }
$$

Sea $\mathrm{P}_{\mathrm{i}}$ igual a uno la probabilidad de que una persona sea pobre dado el conjunto de variables explicativas, entonces en caso contrario, cuando sea cero, la probabilidad será de $1-\mathrm{P}_{1}$, lo que se puede expresar de la siguiente forma:

$$
E\left(Y_{i} \mid X_{i}\right)=0\left(1-P_{i}\right)+1\left(P_{i}\right)=P_{i}
$$

Donde $Y_{i}$ se refiere al evento de ocurrencia de la pobreza y $X_{i}$ el conjunto de variables explicativas.

Las variables dependientes en los modelos serán poblp3 y poblp para México y Chile respectivamente, la primera considera el vector de pobreza patrimonial y la segunda el vector de pobreza (no indigencia) ${ }^{(1)}$. En el caso de la variable poblp3, ésta fue obtenida mediante un conjunto de comandos oficialmente definidos por el Coneval, lo cual está disponible en su portal electrónico. Para el caso de Chile, la variable poblp no se calcula de forma oficial, sin embargo, fue posible obtener el vector de pobreza mediante la encuesta Casen, bajo el criterio de ingreso per cápita del hogar, que se define como la suma del ingreso autónomo (el ingreso por concepto de sueldos y salarios, ganancias provenientes del trabajo independiente, autoprovisión de bienes producidos por el hogar, bonificaciones, gratificaciones, rentas, intereses, así como jubilaciones, pensiones, montepíos y transferencias entre privados), las transferencias monetarias que recibe el hogar del Estado, ambos excluyendo el servicio doméstico puertas adentro, y una imputación por concepto de arriendo de la vivienda, cuando ésta es habitada por sus propietarios (MIDEPLAN, 2012). Las estimaciones del índice de concentración y el modelo Probit de la pobreza se obtendrán con apoyo del software Stata V. 13.

Para describir el fenómeno de pobreza, las variables explicativas del modelo se agrupan en las siguientes categorías como muestra el Cuadro 1.

\section{RESULTADOS}

\section{1 ÍNDICES DE CONCENTRACIÓN DE LOS PTC}

El Cuadro 2 contiene los resultados del índice de concentración. Si el índice es negativo, entonces los programas sociales seleccionados son progresivos; lo que significa que la concentración de los recursos, por concepto de las transferencias, se focaliza en los primeros deciles de ingreso. Las columnas referentes a la concentración de las transferencias denotan la proporción acumulada en los deciles, por ejemplo, el 85.7 y 81.7 por ciento de las transferencias se concentran en los primeros seis deciles de ingreso, para el Programa Oportunidades y Bono de Protección Familiar respectivamente.

(1) Las estimaciones del índice de concentración y el modelo Probit de la pobreza se obtendrán con apoyo del software Stata V. 13. 
El Gráfico 1 muestra la curva de Pseudo-Lorenz para las transferencias de los programas estudiados, que es calculada por el área bajo la curva de concentración y la línea diagonal de 45 grados, la cual se obtendría si existiera igualdad en la distribución (VERAS, 2007). El Programa Oportunidades en México tiene un mayor grado de progresividad, seguido por el Bono de Protección Familiar definido como la herramienta principal del programa Puente y dejando en último puesto el componente de Chile Solidario, Asignación Social.

El Gráfico 1 evidencia la mala distribución de los recursos públicos otorgados a los programas sociales ya que debido al objetivo por el que fueron creados, las líneas que denotan la proporción acumulada por concepto de las transferencias deberían llegar al decil seis como máximo, ya que el nivel de ingreso de una persona pobre se encuentra por debajo de dicho decil. Asimismo, a través del método de líneas de pobreza utilizado en ambos países, la población pobre se determina mediante un determinado nivel de ingreso, que si fuera alto, es decir, si se ubicará en los últimos deciles, no conduciría a que se encontraran en el umbral de la pobreza.

\subsection{RESULTADOS DEL MODELO PROBIT DE LA POBREZA: MÉXICO Y CHILE}

Respecto al modelo de probabilidad obtenido, es necesario mencionar que los parámetros del modelo no son necesariamente los efectos marginales que generalmente se analizan en los modelos lineales, por tal motivo, al estimar el modelo Probit se deben de calcular dichos efectos para que la interpretación de los resultados sea óptima (GREENE, 1998).

Ahora bien, para la significancia individual, se considera el planteamiento, para ambos modelos, de la siguiente hipótesis nula $H_{0}: \beta_{i}=0 \forall i=\{1, \ldots, n\}$ que de aceptarse indicaría la inexistencia de un efecto entre la variable explicativa y, en este caso, el vector de pobreza. Los Cuadros 3 y 4 presentan la probabilidad del estadístico que permite inferir la significancia de las variables a distintos niveles de error ( 5 y 10 por ciento), además del efecto marginal de cada una de las variables explicativas.

De acuerdo con el modelo Probit para el caso mexicano, la probabilidad de ser pobre dado el conjunto de variables explicativas es de 0.7309. Como se puede observar, la variable referente a las transferencias del programa social más importante para el combate contra la pobreza en México es significativa pero el signo que acompaña al coeficiente es positivo, lo que indica que produce un efecto social no deseado. Es decir, Programas tipo Oportunidades son muy limitados en sus alcances hacia el progreso social. Otras variables relevantes, como la educación y una mejor infraestructura de la vivienda, así como un mayor destino de recursos para alimentación y educación producen una disminución de la probabilidad de ser pobre. El efecto anterior ocurre con las variables laborales, por lo que al otorgar oportunidades reales a la población en términos de generación y aseguramiento del empleo es posible reducir la probabilidad de ser pobre en México.

El Cuadro 4 contiene los resultados del modelo Probit de la pobreza para el caso Chileno, en donde la probabilidad de ser pobre dado el conjunto de variables explicativas es de 0.07. Al igual en el caso mexicano, variables como mayores años de educación, acce- 
so a la infraestructura pública y servicios básicos en la vivienda poseen la capacidad de reducir la probabilidad de ocurrencia de la pobreza. Las variables referentes a condición de la ocupación laboral y seguridad del empleo también poseen un efecto inverso en la probabilidad de ser pobre. En forma análoga a lo que ocurre en México, los resultados evidencian que la participación en el Sistema Chile Solidario no produce el efecto deseado en la probabilidad de ocurrencia de la pobreza, situación que ya es reconocida también por el gobierno chileno, que en un comunicado del año 2010 destacó la ineficiencia del programa para la superación de este fenómeno (MIDEPLAN, 2013).

Desde el punto de vista estadístico, uno de los elementos de interpretación en los modelos de probabilidad, que no es particularmente significativo para los modelos con regresada binaria, es la medida de valor de ajuste, que en este caso es un pseudo $R^{2}$ o también llamado $R^{2}$ de McFadden, cuyo valor varía entre 0 y 1 , sin embargo, valores entre 0.2 y 0.4 son altamente aceptados (GUJARATI y PORTER, 2010). Para los modelos de probabilidad calculados, la $R^{2}$ de McFadden fue de 0.2530 para México y el valor máximo alcanzado para Chile, considerado la especificación de las variables explicativas, fue de 0.1477 .

Asimismo, con la finalidad de probar la significancia grupal de las variables independientes, en donde la hipótesis nula hace referencia a que los coeficientes son igual a cero $\left(H_{0}: \beta_{1}=\beta_{2}=\beta_{3} \ldots=\beta_{n} 0\right)$ y cuya interpretación es similar a la prueba individual, se recurre al estadístico de la razón de verosimilitud, el cual sigue la distribución $X^{2}$ con grados de libertad ( $\mathrm{gl}$ ) igual al número de variables explicativas, que es equivalente a la prueba F en el modelo de regresión lineal (Gujarati y Porter, 2010). Partiendo de la probabilidad del estadístico $X^{2}$, que para ambos casos es menor a 0.05 , se concluye que el comportamiento grupal o colectivo de las variables explicativas influye en probabilidad de ser pobre.

Los resultados estadísticos presentados anteriormente muestran la naturaleza de estos programas. Como argumentamos anteriormente, al tratarse de Programas que en el fondo buscaban reemplazar sólidos sistemas de protección social por una mera transferencia monetaria (a la Friedman) el resultado obtenido no hace sino corroborar que en el terreno práctico, la eficiencia de estos programas es muy limitada como estrategia para erradicar la pobreza, ni siquiera llegan a estrategia perfectamente articulada. En realidad estos programas no pueden sustituir ni un amplio Sistema de Protección Social que garantice derechos ciudadanos mínimos ni, mucho menos, una Estrategia Nacional de Desarrollo.

\section{CONCLUSIONES}

Los resultados permiten concluir que los Programas de Transferencias Monetarias Condicionadas no necesariamente deben tener un papel protagónico en el diseño de la política social; ni en particular, en el combate contra la pobreza. Reemplazar la construcción de un amplio y robusto sistema de Protección Social por un ingreso directo a los pobres tiene efectos muy limitados para atender la dimensión social de la pobreza. La política social de México y Chile en las últimas décadas ha tenido como columna 
vertebral la operación de programas sociales que transfieren un monto sujeto a una serie de corresponsabilidades, que si bien, pueden tener ciertos beneficios sobre la población beneficiaria no han resuelto el problema estructural que causa la pobreza. En contrapartida, debería construirse un amplio sistema de protección social que vaya más allá de otorgar un ingreso monetario a los pobres. Se trataría de la construcción de un sistema legal e institucional que permitieran garantizar un amplio con junto de derechos sociales a toda la población.

Diversos elementos son los que han contribuido a que la operación de los programas sociales aquí estudiados no hayan impactado de forma constante a los niveles de pobreza. Bajo el diseño en el que fueron creados, el programa Oportunidades y el Sistema Chile Solidario se concentran principalmente en el mantenimiento de un nivel de ingreso mínimo sujeto a un conjunto de corresponsabilidades en términos de acumulación de capital humano. La modificación de su diseño y operación contribuiría en mayor medida al logro de los objetivos por el que fueron creados.

Los resultados obtenidos confirman que no se puede atribuir a un programa social toda la responsabilidad en la disminución de las cifras de pobreza. En el caso de México, en donde la magnitud de la pobreza es amplia (alcanzando el 50 por ciento de la población y 11 por ciento en pobreza extrema), se obtuvo que la pertenencia al programa Oportunidades no logra cumplir el objetivo del gobierno federal de disminuir la probabilidad de ser pobre. En el caso de Chile, con el Sistema Chile Solidario el resultado fue el mismo. Asimismo, es importante destacar, que si existiera una distribución de las transferencias con mayor grado de progresividad, entonces la probabilidad de ocurrencia de la pobreza sería menor en ambos países.

Por lo anterior y dado que el principal indicador para la medición de la pobreza es el ingreso, es importante implementar una serie de acciones que propicien su generación, que van desde la modificación de los programas sociales base en la política social de México y Chile, hasta la creación y reforzamiento de programas productivos. El enunciado anterior tiene fundamento en los resultados obtenidos en los modelos probit de la pobreza, que definen a la ocupación laboral y aseguramiento del empleo como variables capaces de disminuir la probabilidad de ocurrencia de la misma. Asimismo, los polos sugeridos para el combate contra la pobreza pueden ser conciliados a través de la vinculación entre programas sociales, tanto asistencialistas como productivos, lo que lograría mejores resultados para su superación. Lo anterior se reforzaría además, con una coparticipación del Estado y de las familias beneficiarias para la obtención de un nivel de ingreso que les permita tener acceso a los bienes y servicios básicos que fomenten su bienestar.

\section{BIBLIOGRAFÍA}

\subsection{LIBROS Y REVISTAS}

ANGELUCCI, Manuela; ORAZIO Attanasio; VINCENZO, Di Maro. The impact of Oportunidades on consumption, savings and transfers. Fiscal Studies, v. 33, p. 305-334, 2012. 
ARRIAGADA, Irma; CHARLOTTE, Mathivet. Los programas de alivio a la pobreza Puente y Oportunidades. Una mirada desde los actores. Naciones Unidas, CEPAL, Serie Políticas sociales, n. 134, 2007. BOLTVINIK, Julio; HERNÁNDEZ LAOS, Enrique. Pobreza y distribución del ingreso en México. 2. ed. Siglo Veintiuno Editores, 2000.

BURNS, Eveline. The Beveridge Report. American Economic Review, v. 33, n. 03, pp. 512-533, 1943. CECCHINI, Simone; ALDO, Madariaga. Programas de transferencias condicionadas: balance de la experiencia reciente en América Latina y el Caribe. Naciones Unidas-CEPAL, Cuadernos de la CEPAL, n. 95, 2011.

CECCHINI, Simone; MARTÍNEZ, Rodrigo. Protección social inclusiva en América Latina: una mirada integral, un enfoque de derechos. Naciones Unidas-CEPAL, Libros de la CEPAL, n. 111, 2011.

CETRÉ, Moisés. Pobreza y distribución del ingreso en América Latina, Colombia y Bogotá. Comercio Exterior, v. 56, n. 01, 2006.

DEATON, Angus. The analysis of household surveys: A microeconometric approach to development policy. World Bank, The Johns Hopkins University Press, v. 01, 1997.

FERES, Juan Carlos y MANCERO, Xavier. Enfoques para la medición de la pobreza. Breve revisión de la literatura. Naciones Unidas-CEPAL, Serie estudios estadísticos y prospectivos, n. 4, enero. 2001.

FISZBEIN, Ariel; NORBERT Schady. Conditional cash transfers: reducing present and future poverty. The World Bank, A World Bank Policy Research Report, 2009.

FREDERICKS, Pierce (1978). William Beveridge: A biography. The Journal of Economic History, v. 38, n. 3, Review of the book: Harris, José (1977), William Beveridge: A biography, Oxford University Press, New York.

GREENE, William. Análisis Econométrico. Prentice Hall, 3 ed., 1998.

GUJARATI, Damodar; DAWN C. PORTER. Econometría. 5. ed. Mc Graw Hill, 2010.

HOCES DE LA GUARDIA, Fernando; HOJMAN, Andrés; LARRAÑAGA, Osvaldo. Evaluating the Chile Solidario program, results using the Chile Solidario panel and the administrative databases. Estudios de Economía, v. 38, n. 1, junio 2011.

LEVY, Santiago; RODRÍGUEZ, Evelyne. Sin herencia de pobreza. El programa Progresa-Oportunidades de México. Banco Interamericano de Desarrollo- Editorial Planeta, 1. ed., 2005.

MYLES, John and QUADAGNO, Jill. Political Theories of the Welfare State. Social Service Review, v. 76, n. 1, pp. 34-57, 2002.

PÉREZ, Rafael; VERAS, Fábio; ISSAMU, Guilherme. The impact of CCTs. What we know and what we are not sure about. Poverty in Focus, International Poverty Centre, n. 15, 2008.

RANGEL, Rafael; WERNER, Voigt; AGUILAR, Genaro. La política social en la Unión Europea. En: VILLARESPE, Verónica (coordinadora). Pobreza: concepciones, medición y programas. Universidad Nacional Autónoma de México, Instituto de Investigaciones Económicas, p. 331-362, 2010.

RAVALLION, Martin. Poverty lines in theory and practice. The World Bank, The Living Standards, Measurement Study, working paper n. 133, 1998.

RAVALLION, Martin. Targeted transfers in poor countries: Revisiting the trade-offs and policy options. World Bank, Chronic Poverty Research Centre, working paper n. 26, 2003.

RAWLINGS, Laura; RUBIO, Gloria. Evaluating the impact of conditional cash transfer programs: lessons from Latin America. World Bank Policy Research, working paper n. 3119, 2003.

RIVERA Castiñeira, NUNES, Luis; RUNGO, Paolo. Impacto de los programas de transferencia condicionada de renta: el programa Bolsa Familia de Brasil. Revista Española de Salud Pública, vol. 83, n. 1, enero-febrero 2009. 
SCHAFFLAND, Elke. Conditional Cash Transfers in Brazil: Treatment Evaluation of the "Bolsa Famila" Program on Education. Courant Research Centre: Poverty, Equity and Growth, Discussion Papers, n. 84, 2012.

SOARES, Sergei; GUERREIRO Rafael; VERAS, Fabio; MEDERIOS, Marcelo; ZEPEDA, Eduardo. Conditional Cash Transfers in Brazil, Chile and Mexico: Impacts upon inequality. Estudios Económicos, Número extraordinario, p. 207-224, 2009.

STAMPINI, Marco; TORNAROLLI, Leopoldo. The growth of conditional cash transfers in Latin America and the Caribbean: did they go too far?, Inter-American Development Bank, Social Sector, Social Protection and Health Division, n. 185, noviembre 2012.

VERAS, Fábio; PEREZ, Rafael; GUERREIRO, Rafael. Evaluating the Impact of Brazil's Bolsa Familia: Cash transfer programmes in comparative perspective. International Poverty Centre, n. 01, 2007.

VILLATORO, Pablo. Programa de transferencias monetarias condicionadas: experiencias en América Latina. Revista de la CEPAL, n. 86, pp. 87-101, 2006.

VILLATORO, Pablo. CCTs in Latin America: Human Capital Accumulation and poverty reduction. Poverty in Focus, International Poverty Centre, n. 15, agosto 2008.

WOLMAN, Leo. The Beveridge Report. Political Science Quarterly, v. 58, n. 01, 1943.

\subsection{FUENTES DOCUMENTALES}

BANCO MUNDIAL (BM). Resultados del BIRF. Transferencias monetarias condicionadas parafamilias pobres: Una herramienta eficiente para combatir la pobreza e incrementar el capital humano. Resultados de Proyectos, marzo 2010.

BEVERIDGE, William. Social Insurance and Allied Services, report by Sir William Beveridge, 1942. COMISIÓN ECONÓMICA PARA AMÉRICA LATINA Y EL CARIBE (CEPAL). La protección social de cara al futuro: acceso, financiamiento y solidaridad. Trigésimo primer periodo de sesiones de la Cepal, marzo 2006.

HERNÁNDEZ, María del Carmen. La pobreza en México y análisis de elementos básicos del programa Oportunidades. Tesis de grado para Maestro en Ciencias Económicas, México, Instituto Politécnico Naciona, Escuela Superior de Economía, 2012.

MINISTERIO DE DESARROLLOSOCIAL (MIDEPLAN), Gobierno de Chile. Manual del Investigador Encuesta de Caracterización Socioeconómica Nacional 2011: Módulos Registro, Residentes, Trabajo e Ingresos, 2012.

PLAN NACIONAL DE DESARROLLO DE MÉXICO (PND) 2007-2012.

\subsection{FUENTES ELECTRONICAS Y BASES DE DATOS}

INSTITUTO NACIONAL DEESTADÍSTICA Y GEOGRAFÍA (INEGI). Encuesta Nacional de Ingresos y Gastos de los Hogares 2012, Microdatos.

MINISTERIO DE DESARROLLO SOCIAL (MIDEPLAN), Gobierno de Chile. Portal de internet. Disponible en: <http://www.ministeriodesarrollosocial.gob.cl/>, 2013.

MINISTERIO DE DESARROLLO SOCIAL (MIDEPLAN), Gobierno de Chile. Encuesta de caracterización socioeconómica nacional Casen 2011, Microdatos

MINISTERIO DE DESARROLLO SOCIAL (MIDEPLAN), Encuesta Casen. Portal de internet. Disponible en: <http://observatorio.ministeriodesarrollosocial.gob.cl/index.php>, 2013.

SECRETARÍA DE DESARROLlO SOCIAL (SEDESOL) Gobierno de México. Portal de internet. Disponible en: <www.sedesol.gob.mx>, 2013. 